\title{
Management of tracheal trauma
}

\section{Incidence}

Blunt injury to the lower airway is uncommon, due to anatomic protection by the mandible and sternum anteriorly, the spinal column posteriorly, and the mobility and the elasticity of the upper airway itself. The larynx or cervical trachea is injured in $<1 \%$ of patients admitted to the hospital for blunt trauma. ${ }^{1}$ Over a ten-year period, Angood and co-workers reported that only 16 patients with laryngeal injury and four patients with cervical tracheal injury were treated at the Montreal General Hospital. ${ }^{2}$ Of 46 patients with blunt upper airway injuries reported by Cicala and colleagues, 11 $(24 \%)$ died and four $(36 \%)$ of these deaths were primarily due to airway injury. ${ }^{3}$ Other authors reported that $21 \%$ of patients with blunt airway injuries died in the first two hours after admission. ${ }^{4}$

Penetrating airway injuries in the neck are also relatively uncommon. Capan and colleagues reviewed 17 reports published between 1965-1989 describing acute cervical airway injuries. ${ }^{5}$ Approximately 300 patients were identified accounting for an average of less than three cases per year per reporting centre. Penetrating injuries of the neck involved the larynx in up to $5-15 \%$ of patients, with the patients who had carotid artery or digestive track injuries being at least twice as likely to have airway injuries. ${ }^{6,7}$ About one-third of airway injuries involved the larynx and two-thirds involved the cervical trachea. ${ }^{3}$

The sites of non-penetrating upper airway injuries vary among patients. In a review of a series of blunt injuries to the upper airway, Cicala and co-workers reported seven (35\%) laryngeal injuries above the cricoid, three (15\%) involving the cricoid cartilage, nine $(45 \%)$ involving the cervical trachea, and one $(5 \%)$ involving other sites. ${ }^{3}$ Thyroid cartilage was the most commonly fractured site in blunt and penetrating laryngeal injuries (47\%), followed by arytenoid cartilage (24\%), and cricoid cartilage $(22 \%) .{ }^{8}$ The prevailing site of tracheal transection is the junction of the cricoid with the trachea, because the connective tissues in this area are relatively weak.

\section{Mechanism of injury}

The pattern of injuries is closely related to the mechanism of injury. The majority of laryngotracheal injuries are caused by blunt trauma. Other etiologies include inhalation of noxious or hot gases; aspiration of foreign bodies; iatrogenic injuries; and penetrating trauma.

\section{Inbalation injury}

The inhalation of extremely hot steam, gas, or other noxious fumes will tend to injure the larynx and cervical trachea, and seldom damage the lower airway. The resulting necrosis and scar formation narrows and deforms the affected area. Early intubation or tracheostomy is essential in most cases, due to the rapid development of laryngeal edema.

\section{Intubation injury}

Injuries resulting from endotracheal intubation have been the most common indication for tracheal resection and reconstruction. In a study by Grillo, 208 patients received operations to repair injuries resulting from tracheal intubation; 185 had tracheostomy injuries, and 23 had injuries from endotracheal intubation - with 22 of the 23 being cuff injuries. Cuff injuries also accounted for approximately half of the injuries in the patients who had tracheostomies. The percentage of laryngeal injuries due to intubation is unclear, but a prospective study by Kambic and Radsel reported it to be approximately $0.1 \%{ }^{10}$

The most frequent intubation injury is chronic cicatrix with stenosis. Other injuries include tracheoesophageal fistula, tracheal erosion by the tracheostomy tube, trachea-innominate artery fistula, and bronchial rupture.

High pressures within endotracheal tube cuffs, which were common prior to the introduction of lowpressure high-volume cuffs, are thought to be the etiology of most injuries following endotracheal intubation. Since the development of high-volume low-pressure cuffs, the incidence of tracheal stenosis from this type of injury appears to have decreased, but injuries continue to occur and continue to be the most common indication for tracheal resection and reconstruction. ${ }^{11}$

From the Department of Anesthesia and Critical Care, Massachusetts General Hospital, Boston, Massachusetts, USA.

Address correspondence to: Dr. William E. Hurford, Department of Anesthesia and Critical Care, Massachusetts General Hospital, 55 Fruit Street, Boston, Massachusetts 02114, USA. Phone: 617-726-2859; Fax: 617-724-8511; E-mail: whurford@partners.org 


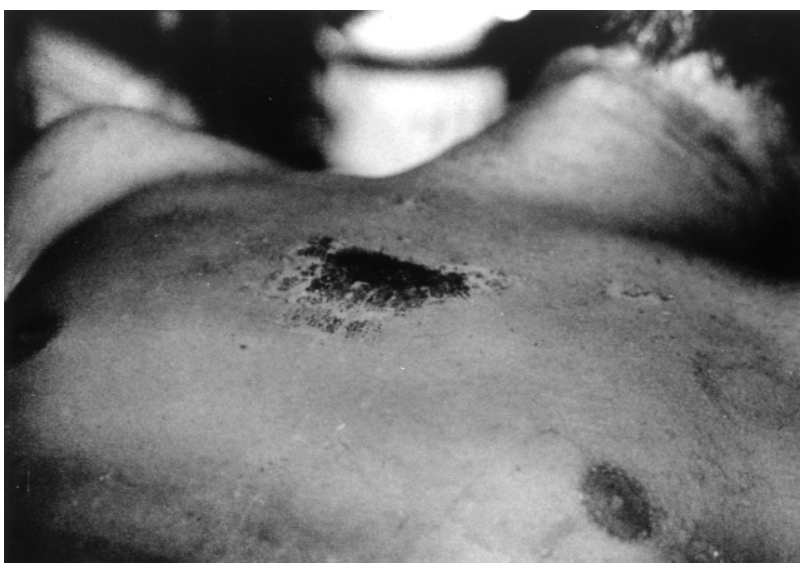

FIGURE 1 Chest contusion and discoloration in an unrestrained driver following a motor vehicle crash. The injury resulted in bronchial disruption at the level of the carina.

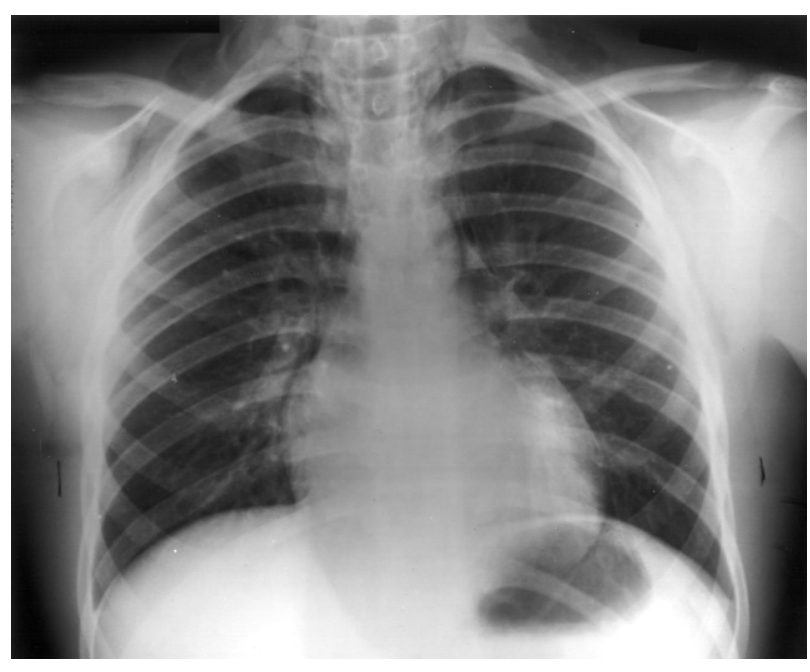

FIGURE 2(A) Tracheal laceration sustained following a dirt bike injury. The chest radiograph demonstrates extensive pneumomediastinum and $\boldsymbol{s} \boldsymbol{c}$ emphysema within the neck.

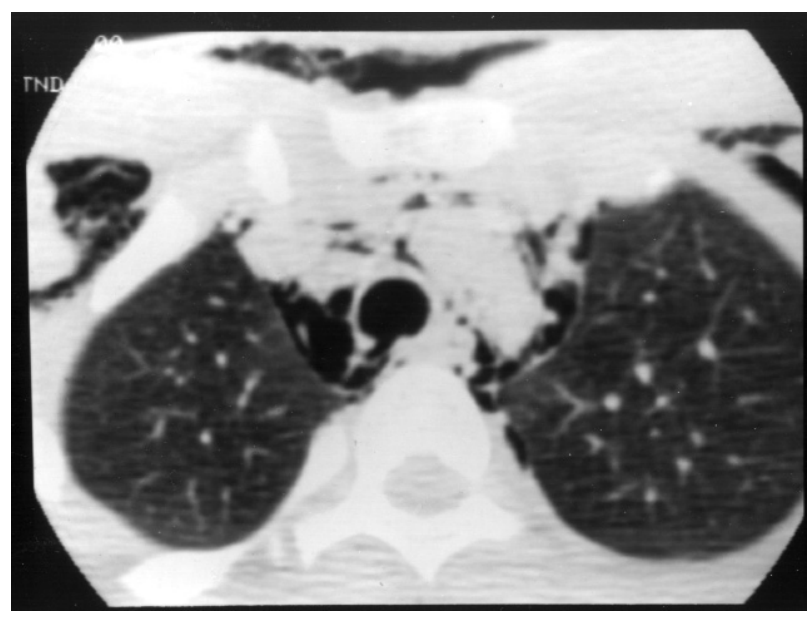

FIGURE 2(B) Axial computed tomography (CT) scan through the superior mediastinum reveals extensive mediastinal emphysema and $\boldsymbol{s} \boldsymbol{c}$ emphysema. There is a laceration of the membranous wall of the trachea.

two-thirds. ${ }^{3}$ Death in patients with penetrating airway trauma is usually due to an associated vascular injury and is rarely due to the airway injury itself. ${ }^{4}$

\section{Associated upper airway pathology}

Edema fluid can rapidly accumulate in the supraglottic and the subglottic submucosa. Subglottic endolaryngeal swelling tends to be circumferential, increasing the possibility of airway obstruction. Air dissecting within with the cervical trachea accounting for the remaining 


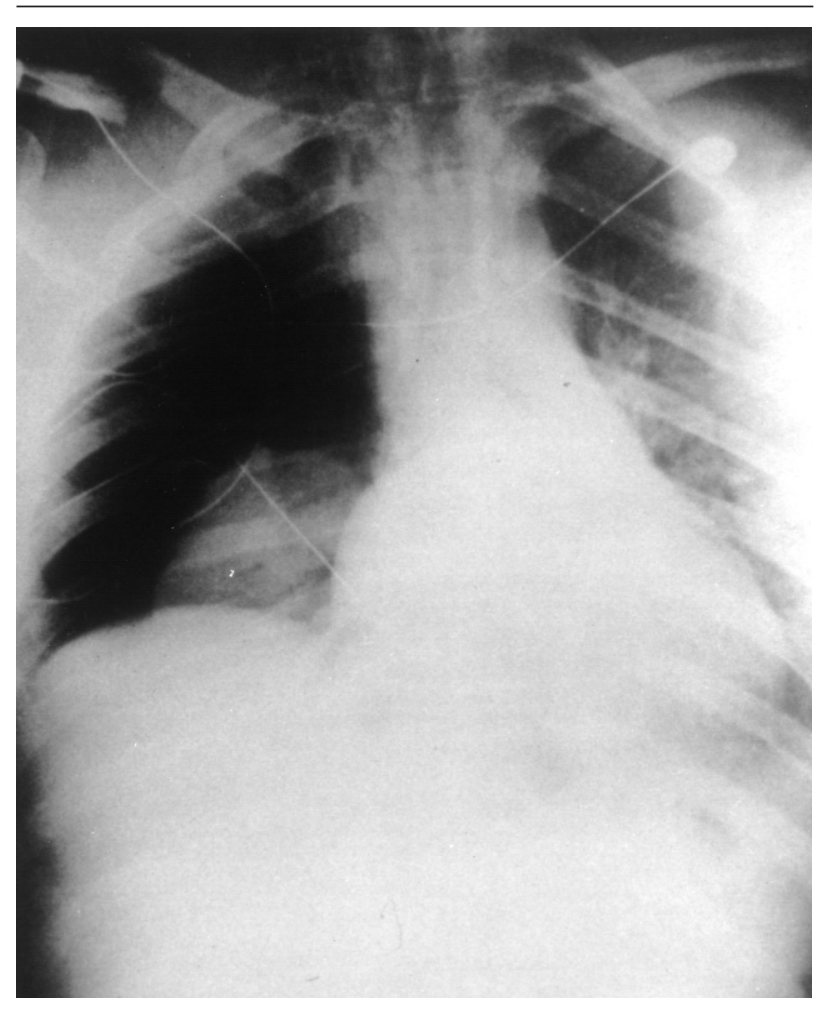

FIGURE 3 Complete avulsion of the right mainstem bronchus, resulting in pneumothorax and complete collapse of the right lung.

the submucosal space can further reduce the luminal diameter of the larynx and trachea. Air within the soft tissues ( $s c$ emphysema) may produce epiglottic emphysema and narrowing of the supraglottic airway. ${ }^{14}$

Most submucosal edema and hematoma formation occurs within several hours after trauma. Consequently, it is unlikely that endolaryngeal swelling will produce airway obstruction more than six hours after the trauma. Straining, coughing, and speaking, however, can increase the amount of $s \boldsymbol{c}$ air and can produce intramural bleeding, which can worsen airway obstruction.

Multiple factors, including the magnitude and direction of force, the position of the cervical spine at impact, the age of the patient, and the consistency of the laryngotracheal cartilages and soft tissue, determine type of airway injury (such as laryngotracheal contusion, edema, hematoma, laceration avulsion, and/or fracture and dislocation of the thyroid, cricoid, or tracheal cartilages) that may occur. ${ }^{5}$ Older patients with calcified cartilages have a higher theoretical risk of laryngeal fracture. However, this theory regarding vulnerability due to age has never been for- mally proved, because patients that sustain this type of injury tend to be younger adults. ${ }^{15} \mathrm{~A}$ combination of soft-tissue, supraglottic, glottic, and infraglottic injuries in a single patient is common.

\section{Associated injuries}

Injuries sufficient to result in severe laryngotracheal damage can also easily damage the cervical spine, esophagus, and vascular structures. At least $10 \%$ and as many $50 \%$ of patients with blunt airway trauma have concurrent cervical spine injuries. ${ }^{2,8}$ A cervical spine injury should be considered present in such injuries until proven otherwise. A cervical injury or neurologic symptoms will determine the timing and surgical management of associated airway injuries. ${ }^{16}$

Patients with blunt laryngotracheal trauma also commonly present with closed head injury. Airway obstruction from the injury can be mistakenly thought to be caused as a result of upper airway obstruction resulting from coma. Maxillofacial injuries are also common and, again, airway obstruction may be blamed on the facial injuries. An emergency cricothyroidotomy performed under such conditions may convert a partial airway obstruction to a complete airway obstruction. Patients with penetrating trauma are likely to have concurrent vascular, esophageal, and thoracic injuries. Esophageal injury occurs in $25 \%$ of patients with penetrating airway injuries, but may be missed until late in the patient's hospital course. ${ }^{4}$

Traumatic asphyxia is a rare syndrome that results from a crush injury, usually of short duration, to the chest. ${ }^{12}$ Associated injuries are expected and include injuries to the chest wall, lung, and liver.

\section{Diagnosis}

\section{Physical findings}

Airway injuries produce a spectrum of signs and symptomatology. Presentation varies according to the mechanism of injury. Reports suggest that at least 25\% of patients with laryngotracheal trauma requiring surgery have no physical evidence of such injury, and may not display symptoms until $24-48 \mathrm{hr}$ after trauma. ${ }^{17}$ In diagnosing laryngotracheal injury, one should take into consideration the type of accident and be alert for signs or a combination of signs of local contusion; sc emphysema; changes in the voice, such as hoarseness or inspiratory stridor; respiratory distress; and hemoptysis. Subcutaneous or mediastinal emphysema are sometimes the only factors present in an injury to the distal trachea, and the airway otherwise appears surprisingly normal. It is imperative that the surgeon, if time and circumstances allow, meticulously evaluate each patient for signs of these injuries. 


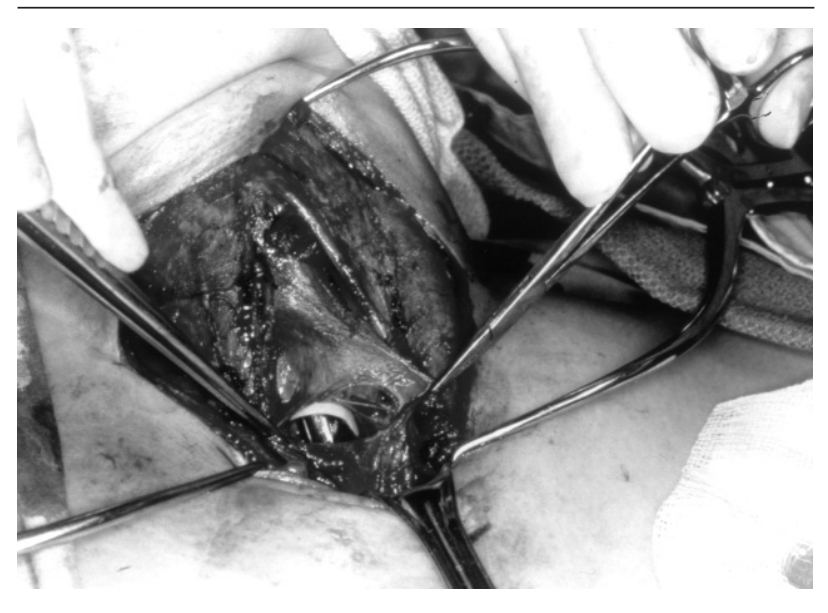

FIGURE 4 Intraoperative view of a tracheal transection. The proximal and distal ends of the trachea remain attached only by the posterior membranous wall and the soft tissues surrounding the trachea. An endotracheal tube is present within the lumen of the trachea.

Injuries to the distal trachea and carina may present with additional findings including $s c$ emphysema and pneumothorax with minimal to a large air leak in which the lung fails to re-expand completely after placement of a chest tube. Laryngoscopy, flexible and rigid bronchoscopy, and esophagoscopy can be performed to definitely delineate the anatomy of the airway and associated injuries.

Failure to recognize injuries acutely may lead to progressive airway obstruction as cicatrization occurs and stenosis develops. The outcome due to delayed diagnosis of associated injuries (i.e., esophageal perforation) can be devastating due to the development of life-threatening complications such as mediastinitis. Delayed diagnosis of laryngotracheal injuries is not unusual, especially when emergency intubation is performed. ${ }^{18}$

\section{Radiological assessment}

Angood and colleagues reported that radiography alone was sufficient for diagnosing $12(60 \%)$ out of 20 patients with cervical airway trauma. ${ }^{2}$ The use of radiographs of the esophagus or cervical spine and additional use of tomograms of the trachea and larynx can be very useful in discovering subtle, previously undetected injuries. Definitive airway management should not be delayed excessively by radiologic studies, since an apparently stable airway can rapidly progress to an acute airway obstruction. Additional radiologic examinations that may be indicated in selected patients include contrast-enhanced esophageal studies, com- puted tomography (CT), and magnetic resonance imaging. High-resolution helical CT scanning can permit three-dimensional reconstruction of the airway. Patients may be unable to lie supine for prolonged radiologic examinations, however, and use of iv sedatives in these patients may lead to disastrous airway compromise and is not advised.

\section{Airway management}

Many patients with upper airway injuries may be successfully managed using traditional techniques to establish an airway. In a retrospective review of upper airway injuries reported by Cicala and co-workers, three-fourths of the patients $(35 / 46)$ had no apparent airway management problems and were either observed without requiring endotracheal intubation (four patients), intubated through an obvious airway defect (six patients), or endotracheally intubated using traditional techniques ( 25 patients). ${ }^{3}$ The remaining patients required emergent tracheostomy, most commonly following blunt trauma.

Airway management in patients with neck trauma is based upon a high index of clinical suspicion for cricoid or cervical tracheal injuries. Attempts at endotracheal intubation in patients with unsuspected cricoid injuries can be disasterous. ${ }^{3}$ Cricoid pressure or the attempted passage of an endotracheal tube may dislocate a fractured cricoid cartilage and/or entirely disrupt a partial tracheal transection, producing complete airway obstruction (Figure 4).

The equipment and personnel required to perform an immediate tracheostomy must be present prior to manipulation of an injured airway. Positive pressure ventilation can exacerbate air leaks and rapidly worsen symptoms from pneumothorax, pneumomediastinum, and air dissecting around airway structures. Whenever possible, the patient should be permitted to breathe spontaneously. Rapid induction of anesthesia and neuromuscular blockade can rapidly produce loss of the airway and the inability to provide positive pressure ventilation. Attempts at direct laryngoscopy or intubation over a flexible bronchoscope may be futile because of bleeding within the airway or distortion of anatomic structures. The danger also exists that flexible bronchoscopy may occlude the airway or precipitate airway obstruction in patients with critical airway stenosis. Under ideal circumstances, pre-oxygenation followed by awake flexible bronchoscopy may permit evaluation of airway injuries and safe endotracheal intubation. Prior induction of general anesthesia, using a potent inhalation anesthetic such as sevoflurane, while maintaining spontaneous ventilation, may be appropriate in some patients. This approach can 


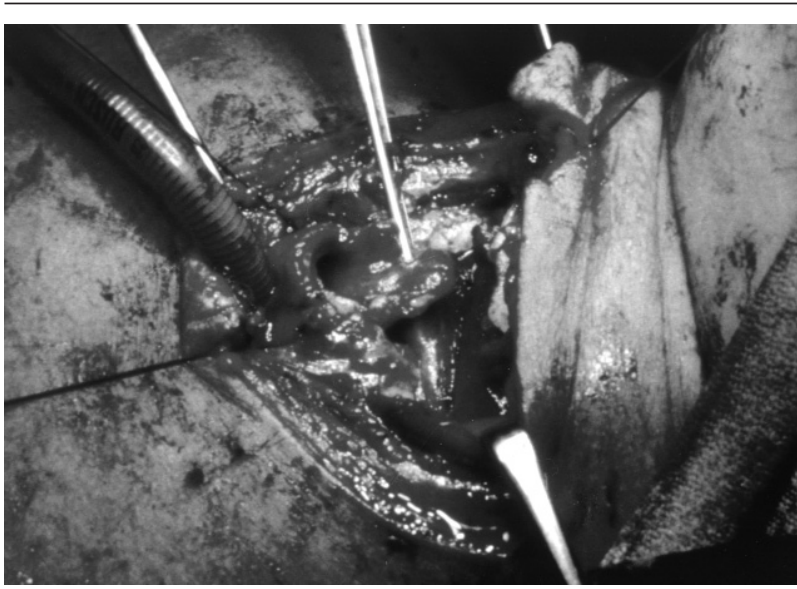

FIGURE 5 Intraoperative view of a complete tracheal transection secondary to a wire "clothesline" injury of the neck. The airway is secured by temporary intubation distal to the site of injury.

permit rigid laryngoscopy and rigid bronchoscopy while maintaining spontaneous ventilation. These techniques may be preferable when bleeding or debris obscure the airway, making fibreoptic examination impossible. If endotracheal intubation appears unwise and the patient is unstable or the airway is lost, immediate tracheostomy is the only appropriate choice. When the trachea itself is injured, it is preferable to conserve normal trachea by placing the tracheostomy through the damaged area. This will facilitate subsequent surgical repair of the trachea.

\section{Anesthetic considerations}

Induction of general anesthesia using a potent volatile agent and spontaneous ventilation is generally considered to be the safest technique to induce anesthesia in patients with possible airway injuries. ${ }^{19}$ The use of iv agents such as propofol, however, may be necessary if the patient is confused or uncooperative. Once anesthetized, the airway may be secured by passing a rigid bronchoscope or an endotracheal tube into the distal airway and past the point of injury. An endobronchial intubation sometimes is necessary to accomplish this. Once an endotracheal tube has been placed across or distal to the site of injury, controlled positive pressure ventilation can begin (Figure 5). The use of neuromuscular blocking agents should generally be avoided until the airway is secured. Positive pressure ventilation by mask may become impossible under such conditions, and may worsen $\boldsymbol{s} \boldsymbol{c}$ emphysema. Immediate tracheostomy is then the only option. Cricothyrotomy may be useless if the cricoid cartilage or distal trachea is injured.

\section{Outcome}

Morbidity

Patients who have suffered severe laryngotracheal injuries usually suffer some permanent airway or voice impairment and may have increased difficulties protecting the airway from aspiration of pharyngeal contents. These complications generally are due to scar contracture or excess granulation tissue formation. Patients sustaining blunt neck trauma tend to have more late complications, such as difficulties with phonation or scarring, than those with penetrating trauma. Schaefer reported that more severe laryngeal injuries are correlated with an increased likelihood and severity long-term sequelae. ${ }^{20}$ Late complications are more common when definitive treatment does not occur for more than $24 \mathrm{hr}$ after injury.

\section{Mortality}

Mortality rates of patients with airway injuries are reported to be between $15 \%-30 \%$, and are usually the result of irreversible shock, massive aspiration of blood, related cervicothoracic vascular injuries, and injured organs. ${ }^{4}$ Emergency management with failed intubation attempts also may produce airway obstruction and even death.

\section{Summary}

Traumatic airway injuries fortunately are rare. Though injuries can be obvious and initial management straightforward, the diagnosis can be difficult. Prompt diagnosis of airway injuries requires a high index of clinical suspicion, complemented by judicious use of endoscopy and radiologic imaging. Initial management can be complicated by associated head, neck, and thoracic injuries. Importantly, a patient's airway can be lost because of injudicious use of sedation or failure to be properly cautious during attempts at airway management and endotracheal intubation. Mortality rates and the incidence of late complications remain high and have been related to delays in diagnosis and definitive treatment.

\section{References}

l Gussack GS, Jurkovich GJ, Luterman A.

Laryngotracheal trauma: a protocol approach to a rare injury. Laryngoscope 1986; 96: 660-5.

2 Angood PB, Attia EL, Brown RA, Mulder DS. Extrinsic civilian trauma to the larynx and cervical trachea-important predictors of long-term morbidity. J Trauma 1986; 26: 869-73.

3 Cicala RS, Kudsk KA, Butts A, Nguyen H, Fabian TC. Initial evaluation and management of upper airway injuries in trauma patients. J Clin Anesth 1991; 3: 91-8. 
4 Kelly JP, Webb WR, Moulder PV, Everson C, Burch BH, Lindsey ES. Management of airway trauma. I: tracheobronchial injuries. Ann Thorac Surg 1985; 40: 551-5.

5 Capan LM, Muller S, Turndorf H. Management of neck injuries. In: Capan LM, Miller S, Turndorf $\mathrm{H}$ (Eds.). Anesthesia and Intensive Care. Philadelphia: JB Lippincott; 1991: 409-46.

6 Asensio JA, Valenziano CP, Falcone RE, Grosh JD. Management of penetrating neck injuries. The controversy surrounding zone II injuries. Surg Clin North Am 1991; 71: 267-96.

7 Miller RH, Duplechain JK. Penetrating wounds of the neck. Otolaryngol Clin North Am 1991; 24: 15-29.

8 Trone TH, Schaefer SD, Carder HM. Blunt and penetrating laryngeal trauma: a 13-year review. Otolaryngol Head Neck Surg 1980; 88: 257-61.

9 Grillo HC. Surgical treatment of postintubation tracheal injuries. J Thorac Cardiovasc Surg 1979; 78: 860-75.

10 Kambic $V$, Radsel $Z$. Intubation lesions of the larynx. Br J Anaesth 1978; 50: 587-90.

11 Grillo HC, Donahue DM. Post intubation tracheal stenosis. Semin Thorac Cardiovasc Surg 1996; 8: 370-80.

12 Sklar DP, Baack B, McFeeley P, Osler T, Marder E, Demarest $G$. Traumatic asphyxia in New Mexico: a fiveyear experience. Am J Emerg Med 1988; 6: 219-23.

13 Richards V, Cohn RB. Rupture of the thoracic trachea and major bronchi following closed injury to the chest. Am J Surg 1955; 90: 253-5.

14 Sacco JJ, Halliday DW. Submucosal epiglottic emphysema complicating bronchial rupture. Anesthesiology 1987; 66: 555-7.

15 Schaefer SD. The treatment of acute external laryngeal injuries. 'State of the art'. Arch Otolaryngol Head Neck Surg 1991; 117: 35-9.

16 Lambert GE Jr, McMurry GT. Laryngotracheal trauma: recognition and management. JACEP 1976; 5: 883-7.

17 Hartmann PK, Mintz G, Verne D, Timen S. Diagnosis and primary management of laryngeal trauma. Oral Surg Oral Med Oral Pathol 1985; 60: 252-7.

18 Couraud L, Velly JF, Martigne C, N'Diaye M. Post traumatic disruption of the laryngo-tracheal junction. Eur J Cardiothorac Surg 1989; 3: 441-4.

19 Flood LM, Astley B. Anaesthetic management of acute laryngeal trauma. Br J Anaesth 1982; 54: 1339-43.

20 Schaefer SD. Primary management of laryngeal trauma. Ann Otol Rhinol Laryngol 1982; 91: 399-402. 